\title{
Resistin Forms A Network With Inflammatory Cytokines And Endothelial Damage Markers And Provides Prognostic And Therapeutic Information For COVID-19
}

\section{Takeshi Ebihara}

Osaka University Graduate School of Medicine https://orcid.org/0000-0003-4101-0419

Hisatake Matsumoto ( $\nabla$ h-matsumoto@hp-emerg.med.osaka-u.ac.jp )

Osaka University Graduate School of Medicine

\section{Tsunehiro Matsubara}

Osaka University Graduate School of Medicine

\section{Yuki Togami}

Osaka University Graduate School of Medicine

\section{Shunichiro Nakao}

Osaka University Graduate School of Medicine

\section{Hiroshi Matsuura}

Osaka Prefecture Nakakawachi Emergency and Critical Care Center

\section{Takashi Kojima}

Osaka University Hospital

\section{Fuminori Sugihara}

Immunology Frontier Research Center and Research Institute for Microbial Desease

\section{Daisuke Okuzaki}

Research Institute for Microbial Disease

Haruhiko Hirata

Osaka University Graduate School of Medicine

Hitoshi Yamamura

Osaka Prefecture Nakakawachi Emergency and Critical Care Center Hiroshi Ogura

Osaka University Graduate School of Medicine

\section{Research}

Keywords: COVID-19, Resistin, Cytokines, Endothelial damage

Posted Date: August 23rd, 2021 
DOI: https://doi.org/10.21203/rs.3.rs-829277/v1

License: (c) (1) This work is licensed under a Creative Commons Attribution 4.0 International License. Read Full License 


\section{Abstract}

Background: Resistin increases in septic subjects and is associated with severity and prognosis. Its role in Coronavirus disease 2019 (COVID-19) is unknown. We investigated relationships between resistin and the severity, prognosis and time to wean off mechanical ventilation (MV) in two cohorts.

Methods: Plasma resistin was available for 306 mild-to-critical COVID-19 patients on days 1, 4 and 8 from the Massachusetts General Hospital Emergency Department COVID-19 (MGH) cohort public proteomics data. The relationship between resistin and severity (World Health Organization COVID-19 outcomes) and the prognosis were evaluated. A cohort of 62 critical COVID-19 patients (Osaka cohort) was used to evaluate the relationship between resistin on days 1 (day of ICU admission), 2-3, 6-8 and 11-15 and the prognosis and time to wean off MV. Correlations among resistin, inflammatory cytokines and endothelial damage markers were evaluated.

Results: In the MGH cohort, day 1 resistin was associated with severity and predicted the prognosis in an ROC analysis (AUC, $0.739 ; 95 \% \mathrm{Cl}, 0.659-0.819$ ). Twenty-eight-day non-survivors showed significantly greater resistin levels than 28-day survivors on days 1, 4 and 8. In the Osaka cohort, a Cox proportional hazards model (time dependent) showed a significant relationship between resistin and time to wean off MV (crude hazard ratio, 0.702 [95\% $\mathrm{Cl}, 0.508-0.969]$ ). Resistin formed a network with inflammatory cytokines and endothelial damage markers.

Conclusions: Resistin was associated with severity, prognosis and time to wean off MV in COVID-19 patients. Resistin formed a network with inflammatory cytokines and endothelial damage markers, suggesting its contribution to the pathogenesis of COVID-19.

\section{Background}

Coronavirus disease 2019 (COVID-19) is a new viral disease caused by severe acute respiratory syndrome coronavirus-2 (SARS-CoV-2), an enveloped RNA beta coronavirus. COVID-19 was first reported in China [1] in December 2019 and rapidly spread globally, infecting over 180,000,000 and causing over $3,800,000$ deaths as of June 25,2021 [2]. The majority of infected individuals exhibit no or mild to moderate symptoms. However, approximately $5-20 \%$ of hospitalized subjects require treatment in an intensive care unit (ICU) because of respiratory failure [3-5]. COVID-19 with respiratory failure requires respiratory support, including invasive mechanical ventilation (MV). Hur et al. reported that $64.1 \%$ of COVID-19 patients with MV were intubated $>14$ days [6]. Nearly $30 \%$ of COVID-19 patients with MV needed a tracheostomy due to prolonged MV [7]. An observational study that evaluated 1,890 COVID-19 patients with tracheostomy in Spain revealed that the tracheostomy was performed a median of 12 days after intubation and that $24 \%$ of these patients remained on MV support at one month after intubation [8]. Prolonged MV management can lead to long-term ICU occupation and the vast use of resources in the ICU. As a result, it could reduce the number of beds that are available for other diseases that usually require ICU management. In fact, an increase in mortality in patients with other diseases was reported 
during the COVID-19 pandemic $[9,10]$. It is important to identify patients with prolonged MV management in addition to those who are likely to die from COVID-19.

In 2001, resistin was first discovered in mice as a hormone released from adipocytes and was reported to be associated with obesity and insulin resistance [11]. In humans, however, resistin seems to be mainly secreted by macrophages rather than adipocytes [12]. Resistin levels are reported to be increased in septic subjects and to be associated with severity and prognosis [13]. Another study reported that resistin is involved in an inflammatory cytokine network that includes interleukin (IL)-6, IL-8, IL-10 and monocyte chemotactic protein (MCP-1) in the acute phase of sepsis and that this network is associated with the severity and prognosis of sepsis [14].

The role of resistin in COVID-19 has remained unclear. Thus, this study aimed to evaluate the relationship between resistin and severity, prognosis and time to wean off MV in COVID-19 patients using two different cohorts.

\section{Methods}

\section{Public proteomics data}

The data used in the present study are publicly available from the Massachusetts General Hospital Emergency Department COVID-19 Cohort (Filbin, Goldberg, Hacohen) with Olink Proteomics (https://www.olink.com/mgh-covid-study/). We named this the MGH COVID-19 cohort. The patients in this cohort were diagnosed as having mild-to-critical COVID-19 according to the definitions of the National Institutes of Health COVID-19 Treatment Guidelines [15]: Mild: varied symptoms but no shortness of breath, dyspnea or abnormal imaging; Severe: $\mathrm{SpO}_{2}<94 \%, \mathrm{PaO}_{2} / \mathrm{FiO}_{2}<300$, respiratory rate $>30$ breaths/minute, or lung infiltration $>50 \%$; and Critical: respiratory failure, septic shock and/or multiorgan dysfunction. The present study was performed in an urban academic hospital in Boston from March 2020 to April 2020. This observational study included 306 COVID-19 patients. The diagnosis of COVID-19 was based on a positive SARS-CoV-2 RT-PCR result. The patients were classified according to their acuity levels (A1-A5) on days 1, 4, 8 and 29 (based on the World Health Organization ordinal outcomes scale [16]: A1, dead; $A 2$, intubated, survived; $A 3$, hospitalized with oxygen; $A 4$, hospitalized without oxygen; $A 5$, discharged). The following clinical data were collected: age, body mass index (BMI),

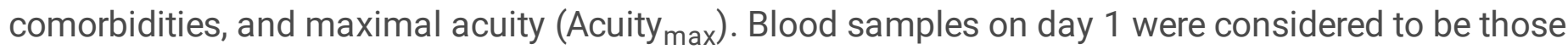
obtained when the initial clinical blood draw was performed in the Emergency Department. Samples were obtained on days 4 and 8 if the patient remained in hospital.

Four panels of proteins (inflammation, oncology, cardiometabolic and neurology proteins) were used to evaluate 1,472 plasma proteins, including 1,463 unique proteins (Olink ${ }^{\circledR}$ Explore 1536). We assessed the relationship between resistin, which was included in the 1,463 proteins, and the prognosis of COVID-19 patients. The Olink multiplex proximity extension assay is a dual-recognition immunoassay. In this assay, two matched antibodies labeled with unique DNA oligonucleotides are used to bind the target proteins. 
Upon binding, these oligonucleotides come into close proximity and hybridize. This is followed by extension, with the generation of a unique sequence used for the digital identification of the specific protein assay (www.olink.com). Plasma $(2.8 \mu \mathrm{l})$ was incubated overnight with oligonucleotide-labeled antibody pairs to form specific DNA duplexes. Extension and pre-amplification were then performed together, and the measurement of individual protein markers was performed using a Nova-Seq 6000 system. These counts were normalized to an extension control and an inter-plate control and then adjusted using a correction factor to calculate the normalized protein expression value (NPX) in log2 scale.

\section{Study design and patient characteristics of the Osaka cohort}

A prospective observational multicenter study of COVID-19 patients was conducted at the Department of Traumatology and Acute Critical Care Medicine, Osaka University Graduate School of Medicine and the Osaka Prefectural Nakakawachi Emergency and Critical Care Center from August 2020 to December 2020. All of the study patients were diagnosed as having SARS CoV-2 (based on a positive RT-PCR test) and pneumonia (based on computed tomography imaging). This group was designated the Osaka COVID-19 cohort. Patients with sepsis who were admitted to the Department of Traumatology and Acute Critical Care Medicine, Osaka University Graduate School of Medicine between February 2014 and July 2015 were included as an ICU control group. The patients with sepsis were all over 18 years of age, and all patients met the Sepsis-3 criteria. Outpatients who were recruited from public poster advertisements were included as a healthy control population.

\section{Clinical data}

Demographic variables (age, sex, BMI), Acute Physiology and Chronic Health Evaluation (APACHE) II score [17] and Sequential Organ Failure Assessment (SOFA) score [18], comorbid conditions (hypertension, diabetes and hyperlipidemia) and clinical variables (day of discontinuation of MV and discharge status) were extracted from electronic medical records by the investigators.

\section{Analysis of resistin, inflammatory cytokines and endothelial damage markers}

ELISAs (R\&D Systems, Minneapolis, MN, USA) were performed to measure the plasma levels of resistin, IL-6, IL-8, IL-10, MCP-1, intercellular adhesion molecule 1 (ICAM-1) and vascular cell adhesion molecule-1 (VCAM-1). After thawing of frozen plasma samples, measurement was conducted according to the manufacturer's protocol. A microplate reader (SH-9000Lab; Corona Electric Co., Ltd., Ibaraki, Japan) was used to measure absorbance. The minimum detectable levels were as follows: resistin, IL-8, IL-10 and ICAM-1: $31.2 \mathrm{pg} / \mathrm{ml}$; MCP-1 and VCAM-1: $15.6 \mathrm{pg} / \mathrm{ml}$; and IL-6: $9.4 \mathrm{pg} / \mathrm{ml}$. 


\section{mRNA expression of resistin in whole blood}

Total RNA was isolated from day 1 leukocyte samples from 10 COVID-19 patients and 5 healthy controls in the Osaka cohort using the PAXgene ${ }^{\text {TM }}$ Blood RNA System (BD Bioscience, San Jose, CA, USA). After blood collection, all collection tubes were stored until further use at $-30^{\circ} \mathrm{C}$. We performed library preparation using a TruSeq stranded mRNA sample prep kit (Illumina, San Diego, CA, USA) according to the manufacturer's instructions. Sequencing was performed with an Illumina NovaSeq 6000 platform in 101-base paired-end mode. Sequenced reads were mapped to the human reference genome sequences (hg19) using the TopHat (version 2.0.13) software program, with Bowtie 2 (version 2.2.3) and SAMtools (version 0.1.19). We calculated the fragments per kilobase of exon per million mapped fragments using the Cufflinks software program (version 2.2.1). FeatureCounts was used to determine the gene-level expression raw read counts. Raw data from this study were submitted for future access under Gene Expression Omnibus accession number GSE179850.

\section{Statistical analysis}

Values are reported as $\mathrm{n}(\%)$ and the median value (quartile 1-3) if the data distribution was skewed, or as the mean \pm SE unless stated otherwise.

In the MGH COVID-19 cohort, the patients were divided into four groups based on the quartiles of the day

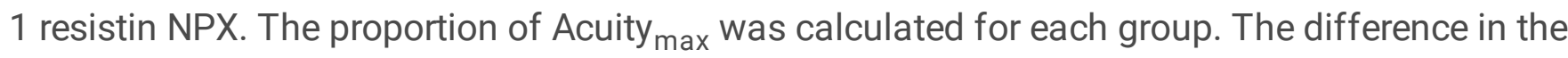
proportion was compared using the chi-squared test. The Wilcoxon rank-sum test was used to evaluate the differences between survivors and non-survivors on day 1 , day 4 and day 8 . To investigate whether the day 1 resistin value is useful as a prognostic biomarker, a receiver operating characteristic (ROC) curve was created. The area under the curve (AUC), accuracy, sensitivity and specificity were also determined.

In the Osaka COVID-19 cohort, resistin, inflammatory cytokines and endothelial damage markers were transformed to logarithmic values to normalize the data distribution before the analyses. Dunnett's test was used to evaluate the difference of each value between patients and healthy controls. Because the median day of weaning off MV was 12 days after intubation in the validation cohort, and that of tracheostomy was 12 days after intubation in a large observational study in Spain [8], treatment with MV for $\leq 12$ days was defined as early recovery, whereas MV $>12$ days and hospital death were defined as late recovery or death, respectively. The patients were divided into two groups in the acute phase (day 1 , days $2-3$, and days $6-8$ ): survivors and non-survivors or early recovery and late recovery or death. Wilcoxon rank-sum tests were used to evaluate differences between two groups on each day. A Cox proportional hazards analysis was performed to estimate hazard ratios and their $95 \%$ confidence intervals (Cls) for the time to wean off MV. The levels of resistin, inflammatory cytokines and endothelial damage markers were analyzed as time-dependent covariates (i.e., the value was updated prospectively for each sample taken). We used the Z score of the log-transformed level in each biomarker to the 
strength of association for comparison among biomarkers. Recognizing that the clinical course may be affected by various factors, we extended this model to adjust for multiple potential confounders, including sex and age, which were found to be associated with the prognosis in COVID-19 [19-21]. We also calculated the median time to wean off $\mathrm{MV}$ in patients divided into two groups according to the quartile of the resistin level determined in a Kaplan-Meier analysis with time as a dependent covariate.

Correlations between resistin, and inflammatory cytokines, and endothelial damage markers were evaluated by a hierarchical clustering analysis based on Spearman correlation coefficients. A network analysis was performed with Cytoscape ${ }^{\circledR}$ software (www.cytoscape.org) version 3.8.0. Log2 fold changes were calculated by dividing the average mediator levels in COVID-19 or sepsis patients by the average levels in healthy controls.

The mRNA expression of resistin was compared between COVID-19 patients and healthy controls by a Wilcoxon rank-sum test.

Statistical analyses were performed using the R software program (version 4.0.2; R Foundation for Statistical Computing, Vienna, Austria). Data are presented using the GraphPad Prism software program (version 8.4.3, GraphPad Software, La Jolla, CA). Statistical significance was defined as $P<0.05$.

\section{Results}

\section{Clinical characteristics and analysis of public proteomics data in MGH COVID-19 cohort}

One of the 306 patient samples in the MGH COVID-19 cohort was identified as an outlier and removed from the final dataset. Accordingly, a total of 305 day 1 samples, 215 day 4 samples and 139 day 8 samples were available in the MGH COVID-19 cohort.

In this cohort, 42 patients died within 28 days and 263 survived until 28 days. Sixty-seven of the survivors received MV. The age and rate of hypertension of the non-survivors were significantly higher than those of the survivors. The rate of diabetes in the two groups did not differ to a statistically significant extent. BMI was not associated with the prognosis (Table 1). 
Table 1

Clinical and demographic characteristics of COVID-19 patients in the MGH cohort

\begin{tabular}{|c|c|c|c|}
\hline & Survivors & Non-survivors & p Value \\
\hline & $(n=263)$ & $(n=42)$ & \\
\hline Age group, $\mathrm{n}(\%)$ & & & $<0.001^{*}$ \\
\hline Under 65 years & $182(69.2)$ & $4(9.5)$ & \\
\hline $65-79$ years & $50(19.0)$ & $15(34.7)$ & \\
\hline Over 80 years & $31(11.8)$ & $23(54.8)$ & \\
\hline BMI group, $\mathrm{n}(\%)$ & & & 0.543 \\
\hline Under 25.0 & $39(14.8)$ & $7(16.7)$ & \\
\hline 25.0-39.9 & $177(67.3)$ & $27(64.3)$ & \\
\hline Over 40.0 & $30(11.4)$ & $5(11.9)$ & \\
\hline Unknown & $17(6.5)$ & $3(7.1)$ & \\
\hline \multicolumn{4}{|c|}{ Comorbidities, n (\%) } \\
\hline Hypertension & $115(43.7)$ & $31(73.8)$ & $<0.001^{*}$ \\
\hline Diabetes & 94 (35.7) & $16(38.1)$ & 0.768 \\
\hline \multicolumn{4}{|c|}{ Significance was determined by Pearson's chi-squared tests } \\
\hline \multicolumn{4}{|c|}{ BMI, body mass index; MGH, Massachusetts General Hospital } \\
\hline \multicolumn{4}{|c|}{ *Statistically significant $(p<0.05)$} \\
\hline
\end{tabular}

The day 1 resistin levels were associated with the 28-day outcome scale values (Fig. 1A). In the COVID-19 patients (days 1, 4 and 8), the resistin levels of the 28-day non-survivors were significantly increased in comparison to those of the 28-day survivors. To explore prognostic biomarkers, an ROC analysis was conducted according to the resistin levels on day 1 and the AUC values were analyzed. The AUC of resistin in the overall COVID-19 patient population was 0.739 (95\% Cl, 0.659-0.819) (Fig. 1C).

\section{Patient characteristics in the Osaka cohort}

The Osaka cohort included 62 COVID-19 patients (male, $n=42$; female, $n=20$ ), 38 patients with sepsis (male, $n=29$; female, $n=9$ ) and 18 healthy controls (male, $n=12$; female, $n=6$ ). All COVID-19 patients were treated in the ICU, and 60 patients $(96.8 \%)$ received MV.

The median APACHE II and SOFA scores in the COVID-19 patients were significantly lower than those in the sepsis patients (14 [9-17] vs. 21 [14-30]; $p<0.001$ and 5 [3-6] vs. 9 [5-13]; $p<0.001)$. The mortality 
rates of the COVID-19 and sepsis patients were $12.9 \%$ and $26.3 \%$, respectively. The comorbidities of the patients are listed in Table 2.

Table 2

Clinical and demographic characteristics of COVID-19 and sepsis patients in the Osaka cohort

\begin{tabular}{|c|c|c|c|}
\hline & Healthy controls & COVID-19 patients & Sepsis patients \\
\hline & $(n=18)$ & $(n=62)$ & $(n=38)$ \\
\hline Age, years & $59(55-71)$ & $71(61-76)$ & $76(65-81)$ \\
\hline Sex, male & $12(66.6)$ & $42(67.7)$ & $29(76.3)$ \\
\hline $\mathrm{BMI}, \mathrm{kg} / \mathrm{m}^{2}$ & $21.6(20.7-25.6)$ & $24.1(22.6-26.3)$ & $21.6(19.0-23.5)$ \\
\hline \multicolumn{4}{|l|}{ Comorbidities } \\
\hline Hypertension & $4(28.5)$ & $33(53.2)$ & $11(28.9)$ \\
\hline Diabetes & $1(7.1)$ & $27(43.5)$ & $15(39.5)$ \\
\hline Hyperlipidemia & $10(55.6)$ & $19(30.6)$ & $7(18.4)$ \\
\hline APACHE II score & & $14(9-17)$ & $21(14-30)$ \\
\hline SOFA score & & $5(3-6)$ & $9(5-13)$ \\
\hline Hospital death & & 8 (12.9) & $10(26.3)$ \\
\hline \multicolumn{4}{|c|}{ Note: Data are given as the median (25th-75th percentile) or as number (\%) } \\
\hline \multicolumn{4}{|c|}{$\begin{array}{l}\text { IQR, interquartile range; BMI, body mass index; APACHE, Acute Physiologic Assessment and Chroni } \\
\text { Health Evaluation; SOFA, sequential organ failure assessment }\end{array}$} \\
\hline
\end{tabular}

In comparison to those of the healthy controls, the plasma resistin levels of both the COVID-19 and sepsis patients in the Osaka cohort were significantly higher on day 1 , days $2-3$, days $6-8$ and days $11-15$. The resistin levels of sepsis patients were significantly higher in comparison to those of the COVID-19 patients (Fig. 2A). Resistin levels were also evaluated to investigate differences between survivors and non-survivors during the acute phase (day 1 , days $2-3$ and days 6-8) in the COVID-19 and sepsis patients (Fig. 2B). There were no differences in the plasma resistin levels of the survivors and the nonsurvivors in the COVID-19 group. However, the plasma resistin levels of the non-survivors were significantly higher than those of the survivors in the sepsis group from day 1 until days $6-8$. Changes in cytokines and endothelial damage markers are shown in Figures S1 and S2 in the online data supplement. 
In a Cox proportional hazards analysis with time-dependent covariates, the resistin level showed a statistically significant association with the time to wean off MV (Table 3). Inflammatory cytokines and endothelial damage markers are also evaluated in Table S1 in the online data supplement. The median time to wean off MV was significantly shorter in patients who had a resistin level in the first quartile $(2018 \mathrm{pg} / \mathrm{ml})$ and lower in comparison to those with higher resistin levels (8 days vs. 15 days in median, $p=0.02$; Figure $S 3$ in the online data supplement). Regarding the outcomes, the plasma resistin levels in patients with late recovery and death were significantly higher in comparison to those with early recovery on days 6-8 (Fig. 3A). Decreased plasma resistin levels were observed in the patients in the early recovery group but not in patients of in the recovery and death group during the acute phase (Fig. 3B).

Table 3

Cox proportional hazard analysis with time as the dependent covariate for weaning off MV

\begin{tabular}{|c|c|c|c|c|c|c|}
\hline & Crude HR & $95 \% \mathrm{Cl}$ & $P$ value & Adjusted HR & $95 \% \mathrm{Cl}$ & $P$ value \\
\hline Resistin & 0.702 & 0.508 to 0.969 & 0.03 & 0.649 & 0.471 to 0.894 & $<0.01$ \\
\hline
\end{tabular}

\section{Network analysis of resistin, VCAM-1, ICAM-1, IL-6, IL-8, IL- 10 and MCP-1 in the Osaka cohort}

The networks of resistin, IL-6, IL-8, IL-10, VCAM- 1 and ICAM-1 on day 1 , days $2-3$, and days $6-8$ in the Osaka COVID-19 and sepsis patients are depicted in Fig. 4. Through the acute phase, resistin was significantly associated with IL-6, IL-8, IL-10, VCAM-1 and ICAM- 1 in the COVID-19 patients, and with IL-6, IL-8 and VCAM- 1 in the sepsis patients.

\section{mRNA expression of resistin in whole blood in the Osaka cohort}

The mRNA expression of resistin in whole blood in the COVID-19 patients of the Osaka cohort was significantly higher in comparison to that in the controls (Figure S4 in the online data supplement).

\section{Discussion}

This is the first study, to our knowledge, to identify a relationship between resistin and severity, prognosis and the time to wean off MV in COVID-19 patients. In addition to sepsis patients, resistin-which was elevated in COVID-19 patients in comparison to healthy subjects-formed a network with cytokines and endothelial damage markers, which may be involved in the pathogenesis of the COVID-19. Thus, resistin may be a clinical useful biomarker in COVID-19. 
In humans, resistin is delivered from peripheral blood mononuclear cells, macrophages and bone marrow, rather than from adipocytes $[12,22,23]$. In the present study, the resistin gene expression in whole blood cells was elevated, suggesting that these cells were responsible for the production of resistin in COVID-19 patients. Resistin is reported to play a role as a pro-inflammatory cytokine [24] and to be related to the pathogeneses of cardiovascular disease [25], cancer [26] and sepsis [13, 14, 27]. In the present study, COVID-19 patients showed increased plasma resistin levels (although these levels were lower than those in sepsis patients) and resistin, cytokines and endothelial damage markers formed a network.

Resistin has two potential roles in the pathogenesis of COVID-19. First, resistin induces various inflammatory cytokines. In vitro, resistin has been shown to induce the nuclear translocation of NF-kB transcription factors in macrophages and to lead to the increased expression of several pro-inflammatory cytokines, including IL-1, IL-6, IL-12 and TNF- $a$, in both mice and humans [28]. In a study of sepsis patients, resistin was reported to compose a strong network with IL-6, IL-8 and MCP-1 during the acute phase, and that network was reported to be related to the severity and prognosis of sepsis [14]. Second, resistin directly activates vascular endothelial cells. Resistin induced aggravation of an inflammatory condition in the vessel walls of rabbits by stimulating monocytes to infiltrate endothelial cells [29], and it stimulated adhesion of human monocyte cells to human vascular endothelial cells [30]. Clinically, resistin was reported to be associated with endothelial damage markers, including ICAM- 1 and VCAM- 1 in sepsis [31]. The network with resistin, inflammatory cytokines and endothelial damage markers in COVID-19 shown in this study is similar to that observed in sepsis. The action of resistin may be a common response to infection that is pathogen independent. In the MGH COVID-19 cohort, which included mild-tocritical patients, resistin was associated with severity and prognosis, suggesting that the systemic inflammation and endothelial damage caused by the resistin-related network might contribute to a lethal outcome in COVID-19 patients.

A previous study showed that COVID-19 patients often needed prolonged MV management with tracheostomy [8]. In the present study, 60 COVID-19 patients received MV management. The median time of weaning off MV was 12 days after intubation and $25 \%$ of the patients required MV management for > 28 days $(n=12)$. A gradual decrease in the resistin level was observed in the early recovery group. The resistin level had a relationship with the time to wean off MV. The median time to wean off MV was significantly shorter in patients who had a resistin level in the first quartile $(2018 \mathrm{pg} / \mathrm{ml})$ or lower in comparison to those with higher resistin levels (median 8 days vs. 15 days, $p=0.02$ ). Resistin may be useful as a marker to predict the time to wean off MV.

Many biomarkers have been reported in COVID-19 [32], especially IL-6 [33-35]. IL-6 receptor blockade has been investigated for potential therapeutic application [36, 37]. In the present study, which only included critical COVID-19 patients, IL-6 was not shown to be associated with the prognosis or the time to wean off MV. In contrast, resistin was a useful biomarker that predicted severity and prognosis in the mild-tocritical COVID-19 cohort. Furthermore, in the critical patient cohort, resistin was a remarkable biomarker for predicting the time to wean off MV. 
The present study was associated with several limitations. First, the Osaka cohort only included critical COVID-19 patients who were treated in a critical care center. Consequently, this cohort did not represent the entire COVID-19 population. Second, the study population was relatively small.

\section{Conclusion}

Resistin levels were assessed over time in patients with COVID-19. We found that resistin levels were associated with severity, prognosis and the time to wean off MV. Resistin formed a network with inflammatory cytokines and endothelial damage markers, suggesting its contribution to the pathogenesis of COVID-19.

\section{Abbreviations}

APACHE

Acute Physiology and Chronic Health Evaluation; AUC:Area under the curve; BMI:Body mass index; Cl:Confidence interval; COVID-19:Coronavirus disease 2019; ICAM-1:Intercellular adhesion molecule 1; ICU:Intensive care unit; IL:Interleukin; MCP-1:Monocyte chemotactic protein 1; MGH:Massachusetts General Hospital; MV:Mechanical ventilation; NPX:Normalized protein expression value; ROC:Receiver operating characteristic; SARS-CoV-2:Severe acute respiratory syndrome coronavirus-2; SOFA:Sequential Organ Failure Assessment; VCAM-1:vascular cell adhesion molecule-1.

\section{Declarations}

\section{Ethics approval and consent to participate}

This study was performed according to the principles of the Declaration of Helsinki and received approval from the institutional review board of Osaka University Hospital (Permit Numbers: 12007, 16109 and 885 [Osaka University Critical Care Consortium Novel Omix Project; Occonomix Project]). Informed consent was obtained from all patients or their relatives, and the healthy volunteers gave their informed consent for the collection of their blood samples.

\section{Consent for publication}

Not applicable

\section{Availability of data and material}

The raw data have been deposited to Gene Expression Omnibus under accession no. GSE179850.

\section{Competing interests}

The authors declare that they have no competing interests in association with the present study. 


\section{Funding}

This study was supported by JSPS KAKENHI Grant Number $20 \mathrm{~K} 17892$ and by the Japan Agency for Medical Research and Development Grant Number 20fk0108404h0001.

\section{Authors' contributions}

TE conceived and designed this study, acquired the data, and analyzed and wrote the manuscript. Hisatake $\mathrm{M}$ helped with designing the study and data interpretation and conducted the literature review. TM, YT, TK, Hiroshi M, HH and HY contributed to acquiring the data. FS and DO helped analyze the data. SN helped with designing the study. $\mathrm{HO}$ conducted the literature review. All authors have read and understood the journal's policies and believe that neither the manuscript nor the study violates any of these. All authors meet the authorship criteria detailed in the submission guidelines, and all authors agree with the content of the manuscript.

\section{Acknowledgements}

We greatly appreciate the patients, family members and healthy volunteers involved in this study. We would also like to thank all medical staff who cooperated with this study.

\section{References}

1. Coronaviridae Study Group of the International Committee on Taxonomy of Viruses. The species Severe acute respiratory syndrome-related coronavirus: classifying 2019-nCoV and naming it SARSCoV-2. Nat Microbiol. 2020;5:536-44.

2. COVID-19 Map. Johns Hopkins Coronavirus Resource Center. https://coronavirus.jhu.edu/map.html. Accessed 25 Jun 2021.

3. Guan WJ, Ni ZY, Hu Y, Liang WH, Ou CQ, He JX, et al. China Medical Treatment Expert Group for Covid-19. Clinical characteristics of coronavirus disease 2019 in China. N Engl J Med. 2020;382:1708-20.

4. Chen N, Zhou M, Dong X, Qu J, Gong F, Han Y, et al. Epidemiological and clinical characteristics of 99 cases of 2019 novel coronavirus pneumonia in Wuhan, China: A descriptive study. Lancet. 2020;395:507-13.

5. Richardson S, Hirsch JS, Narasimhan M, Crawford JM, McGinn T, Davidson KW, et al. Presenting characteristics, comorbidities, and outcomes among 5700 patients hospitalized with COVID-19 in the New York City area. JAMA. 2020;323:2052-9.

6. Hur K, Price CPE, Gray EL, Gulati RK, Maksimoski M, Racette SD, et al. Factors associated with Intubation and prolonged intubation in hospitalized patients with COVID-19. Otolaryngol Head Neck Surg. 2020;163:170-8.

7. Sancho J, Ferrer S, Lahosa C, Posadas T, Bures E, Bañuls P, et al. Tracheostomy in patients with COVID-19: Predictors and clinical features. Eur Arch Otorhinolaryngol. 2021;1-9. 
doi:10.1007/s00405-020-06555-x (In press).

8. Martin-Villares C, Perez Molina-Ramirez C, Bartolome-Benito M, Bernal-Sprekelsen M, COVID ORL ESP Collaborative Group $\left(^{*}\right)$. Outcome of 1890 tracheostomies for critical COVID-19 patients: a national cohort study in Spain. Eur Arch Otorhinolaryngol. 2020;278:1605-12.

9. Bodilsen J, Nielsen PB, Søgaard M, Dalager-Pedersen M, Speiser LOZ, Yndigegn T, et al. Hospital admission and mortality rates for non-covid diseases in Denmark during covid-19 pandemic: Nationwide population based cohort study. BMJ. 2021;373:n1135.

10. Kursumovic E, Cook TM, Vindrola-Padros C, Kane AD, Armstrong RA, Waite O, et al. The impact of COVID-19 on anaesthesia and critical care services in the UK: a serial service evaluation. Anaesthesia. 2021;76(9):1167-75. doi:10.1111/anae.15512 (In press).

11. Steppan CM, Lazar MA. Resistin and obesity-associated insulin resistance. Trends Endocrinol Metab. 2002;13:18-23.

12. Patel L, Buckels AC, Kinghorn IJ, Murdock PR, Holbrook JD, Plumpton C, et al. Resistin is expressed in human macrophages and directly regulated by PPARY activators. Biochem Biophys Res Commun. 2003;300:472-6.

13. Koch A, Gressner OA, Sanson E, Tacke F, Trautwein C. Serum resistin levels in critically ill patients are associated with inflammation, organ dysfunction and metabolism and may predict survival of nonseptic patients. Crit Care. 2009;13:R95.

14. Ebihara T, Matsumoto H, Matsubara T, Matsuura H, Hirose T, Shimizu K, et al. Adipocytokine profile reveals resistin forming a prognostic-related cytokine network in the acute phase of sepsis. Shock. 2021. doi:10.1097/SHK.0000000000001756 (In press).

15. Information on COVID-19 Treatment, Prevention and Research. COVID-19 Treatment Guidelines. https://www.covid19treatmentguidelines.nih.gov/. Accessed 5 Jul 2021.

16. COVID-19 Therapeutic Trial Synopsis. https://www.who.int/publications-detail-redirect/covid-19therapeutic-trial-synopsis. Accessed 5 Jul 2021.

17. Knaus WA, Draper EA, Wagner DP, Zimmerman JE. APACHE II: A severity of disease classification system. Crit Care Med. 1985;13:818-29.

18. Janssens U, Dujardin R, Graf J, Lepper W, Ortlepp J, Merx M, et al. Value of SOFA (Sequential Organ Failure Assessment) score and total maximum SOFA score in 812 patients with acute cardiovascular disorders. Crit Care. 2001;5:P225.

19. Docherty AB, Harrison EM, Green CA, Hardwick HE, Pius R, Norman L, et al. Features of 20133 UK patients in hospital with covid-19 using the ISARIC WHO Clinical Characterisation Protocol: Prospective observational cohort study. BMJ. 2020;369:m1985.

20. Rapp JL, Lieberman-Cribbin W, Tuminello S, Taioli E. Male Sex, severe obesity, older age, and chronic kidney disease are associated with COVID-19 severity and mortality in New York City. Chest. 2021;159:112-5.

21. Dehingia N, Raj A. Sex differences in COVID-19 case fatality: Do we know enough? Lancet Glob Health. 2021;9:e14-5. 
22. Lin Q, Price SA, Skinner JT, Hu B, Fan C, Yamaji-Kegan K, et al. Systemic evaluation and localization of resistin expression in normal human tissues by a newly developed monoclonal antibody. PLoS One. 2020;15:e0235546.

23. Savage DB, Sewter CP, Klenk ES, Segal DG, Vidal-Puig A, Considine RV, et al. Resistin / Fizz3 expression in relation to obesity and peroxisome proliferator-activated receptor-gamma action in humans. Diabetes. 2001;50:2199-202.

24. Jamaluddin MS, Weakley SM, Yao Q, Chen C. Resistin: functional roles and therapeutic considerations for cardiovascular disease. Br J Pharmacol. 2012;165:622-32.

25. Zhang JZ, Gao Y, Zheng YY, Liu F, Yang YN, Li XM, et al. Increased serum resistin level is associated with coronary heart disease. Oncotarget. 2017;8:50148-54.

26. Sudan SK, Deshmukh SK, Poosarla T, Holliday NP, Dyess DL, Singh AP, et al. Resistin: an inflammatory cytokine with multi-faceted roles in cancer. Biochim Biophys Acta Rev Cancer. 2020;1874:188419.

27. Vassiliadi DA, Tzanela M, Kotanidou A, Orfanos SE, Nikitas N, Armaganidis A, et al. Serial changes in adiponectin and resistin in critically ill patients with sepsis: Associations with sepsis phase, severity, and circulating cytokine levels. J Crit Care. 2012;27:400-9.

28. Silswal N, Singh AK, Aruna B, Mukhopadhyay S, Ghosh S, Ehtesham NZ. Human resistin stimulates the pro-inflammatory cytokines TNF- $a$ and IL-12 in macrophages by NF-KB-dependent pathway. Biochem Biophys Res Commun. 2005;334:1092-101.

29. Cho Y, Lee SE, Lee HC, Hur J, Lee S, Youn SW, et al. Adipokine resistin is a key player to modulate monocytes, endothelial Cells, and smooth muscle cells, leading to progression of atherosclerosis in rabbit carotid artery. J Am Coll Cardiol. 2011;57:99-109.

30. Hsu WY, Chao YW, Tsai YL, Lien CC, Chang CF, Deng MC, et al. Resistin induces monocyteendothelial cell adhesion by increasing ICAM-1 and VCAM-1 expression in endothelial cells via p38MAPK-dependent pathway. J Cell Physiol. 2011;226:2181-8.

31. Macdonald SPJ, Bosio E, Neil C, Arendts G, Burrows S, Smart L, et al. Resistin and NGAL are associated with inflammatory response, endothelial activation and clinical outcomes in sepsis. Inflamm Res. 2017;66:611-9.

32. Gao YD, Ding M, Dong X, Zhang JJ, Kursat Azkur A, Azkur D, et al. Risk factors for severe and critically ill COVID-19 patients: A review. Allergy. 2021;76:428-55.

33. Herold T, Jurinovic V, Arnreich C, Lipworth BJ, Hellmuth JC, von Bergwelt-Baildon M, et al. Elevated levels of IL- 6 and CRP predict the need for mechanical ventilation in COVID-19. J Allergy Clin Immunol. 2020;146:128-36.e4.

34. Laguna-Goya R, Utrero-Rico A, Talayero P, Lasa-Lazaro M, Ramirez-Fernandez A, Naranjo L, et al. IL-6based mortality risk model for hospitalized patients with COVID-19. J Allergy Clin Immunol. 2020;146:799-807.e9.

35. Liu T, Zhang J, Yang Y, Ma H, Li Z, Zhang J, et al. The role of interleukin- 6 in monitoring severe case of coronavirus disease 2019. EMBO Mol Med. 2020;12:e12421. 
36. Salama C, Han J, Yau L, Reiss WG, Kramer B, Neidhart JD, et al. Tocilizumab in patients hospitalized with Covid-19 pneumonia. N Engl J Med. 2021;384:20-30.

37. Stone JH, Frigault MJ, Serling-Boyd NJ, Fernandes AD, Harvey L, Foulkes AS, et al. Efficacy of tocilizumab in patients hospitalized with Covid-19. N Engl J Med. 2020;383:2333-44.

\section{Figures}

(A)

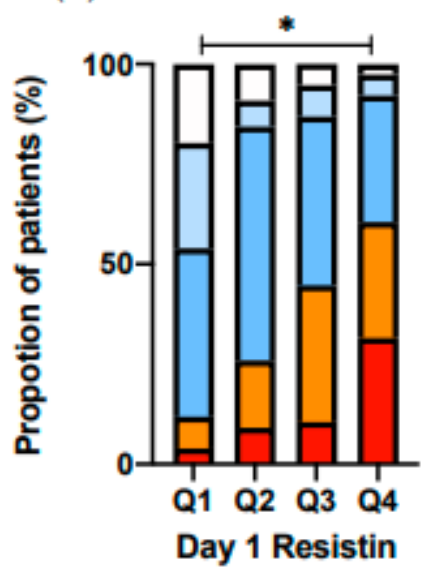

$\square$ Not hospitalized

$\square$ Hospitalized, no supplemental $\mathrm{O}_{2}$ required

$\square$ Hospitalized, supplemental $\mathrm{O}_{2}$ required

$\square$ Intubated, ventilated, survived to 28 days

$\square$ Death within 28 days

(B)

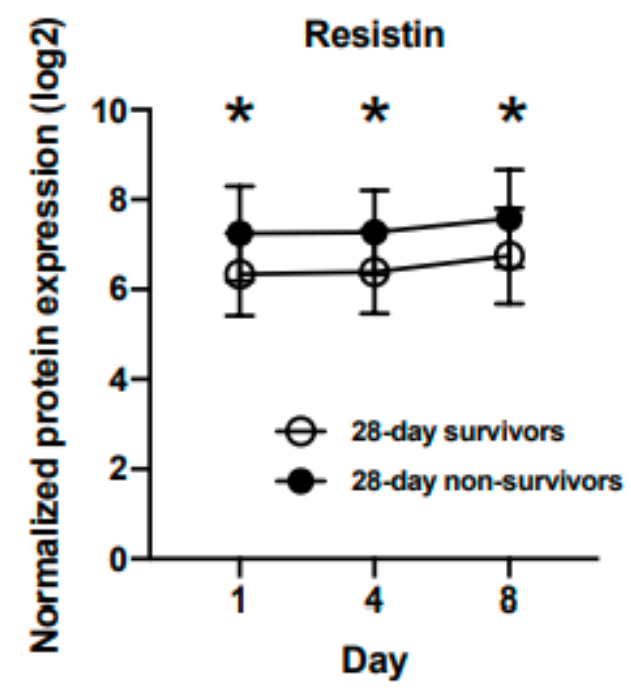

(C)

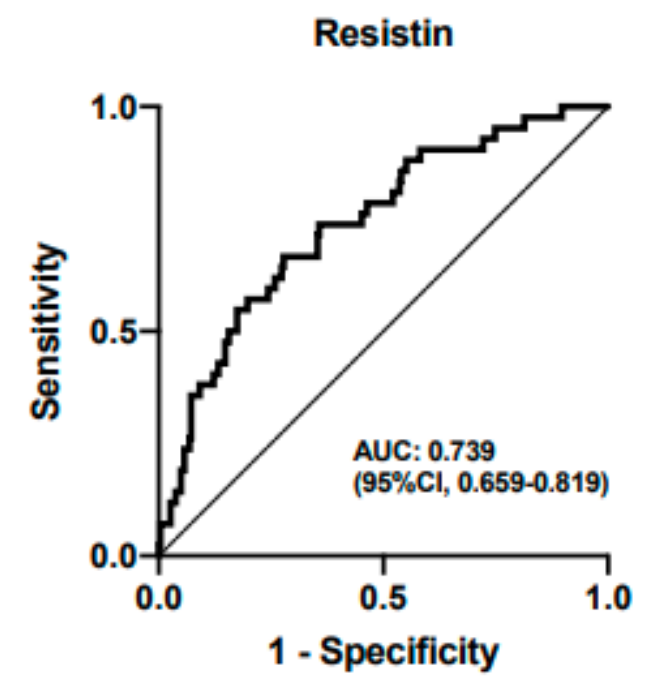

\section{Figure 1}

The evaluation of resistin in the public proteomics data of the MGH COVID-19 cohort. (A) The clinical course by quartiles of day 1 resistin levels. Asterisk indicates a statistically significant difference between the clinical course and the day 1 resistin levels. (B) Change in the NPX of resistin for 28-day survivors and non-survivor on day 1 , day 4 , and day 8 . Asterisk indicates a statistically significant difference between survivors and non-survivors $(p<0.05)$ on each day. (C) The NPX of resistin on day 1 was included in an 
ROC curve analysis, and the AUC was calculated to evaluate the prognostic accuracy. COVID-19, coronavirus disease 2019; NPX, normalized protein expression value; ROC, receiver operating characteristic; AUC, area under the curve

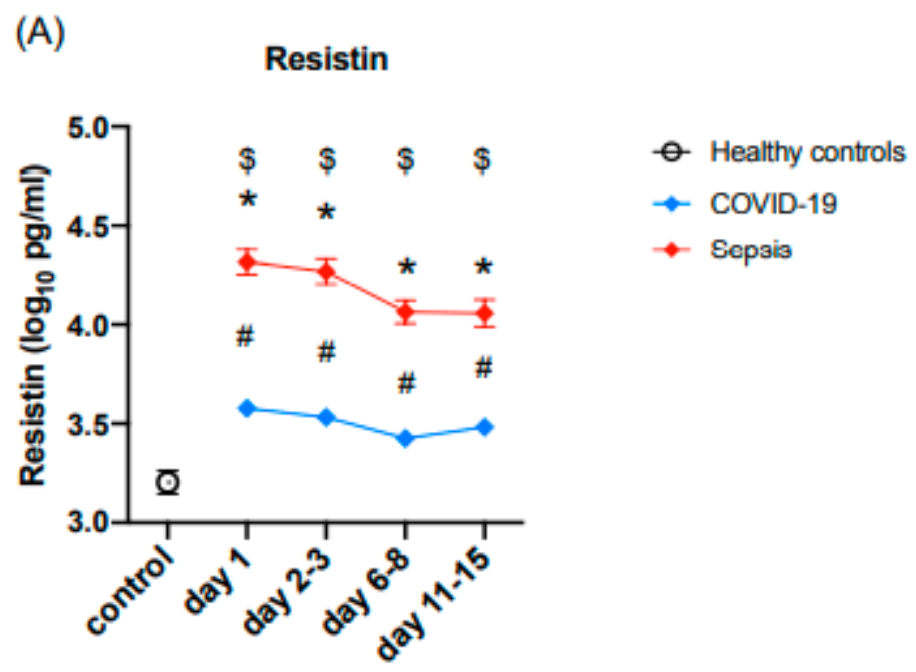

(B)
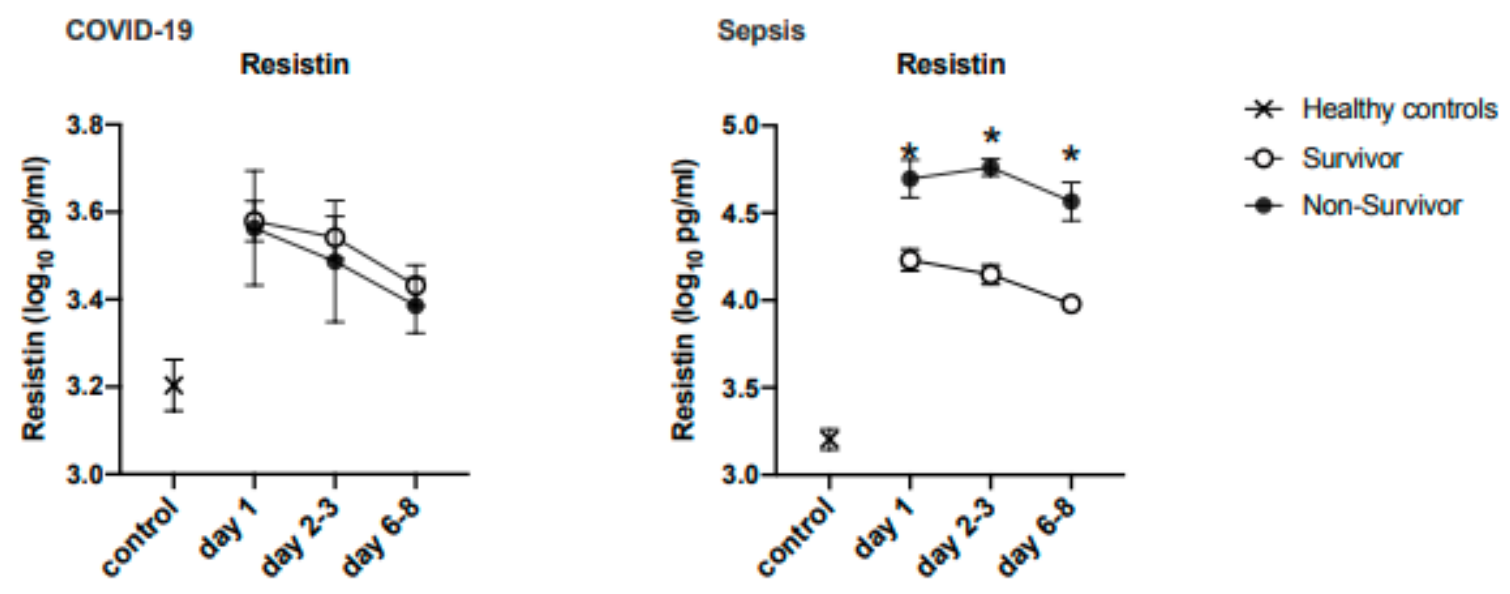

Figure 2

Change in the resistin levels of the Osaka cohort. The resistin levels were transformed to common logarithmic values to normalize the data distribution. All values are expressed as the mean $\pm S E$. (A) * indicates a significant difference in resistin between the control and sepsis patients. \# indicates a statistically significant difference between the control and COVID-19 patients on each day $(p<0.05)$. $\$$ indicates a statistically significant difference between the sepsis patients and COVID-19 patients. (B) The resistin levels of survivors and non-survivors on each day in the sepsis and COVID-19 groups. * indicates a statistically significant difference between survivors and non-survivors $(p<0.05)$ on each day. SE, standard error; COVID-19, coronavirus disease 2019 
(A)

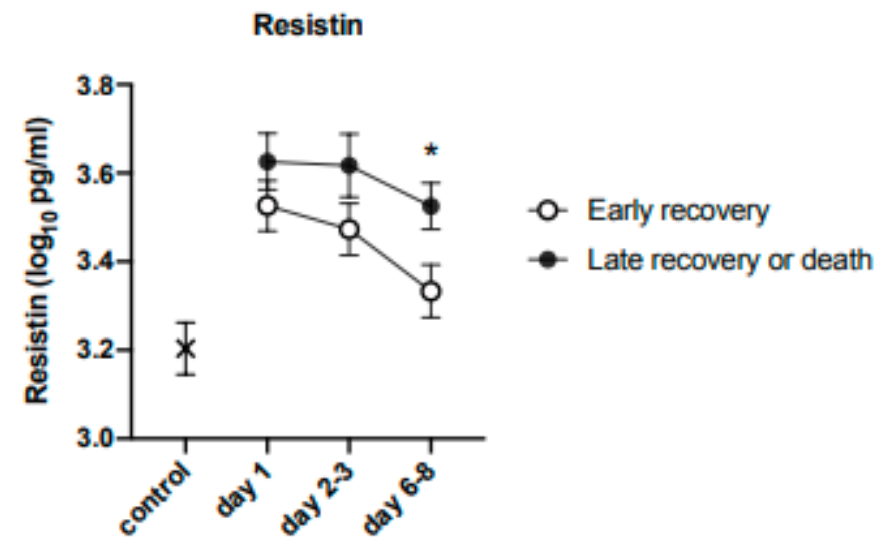

(B)

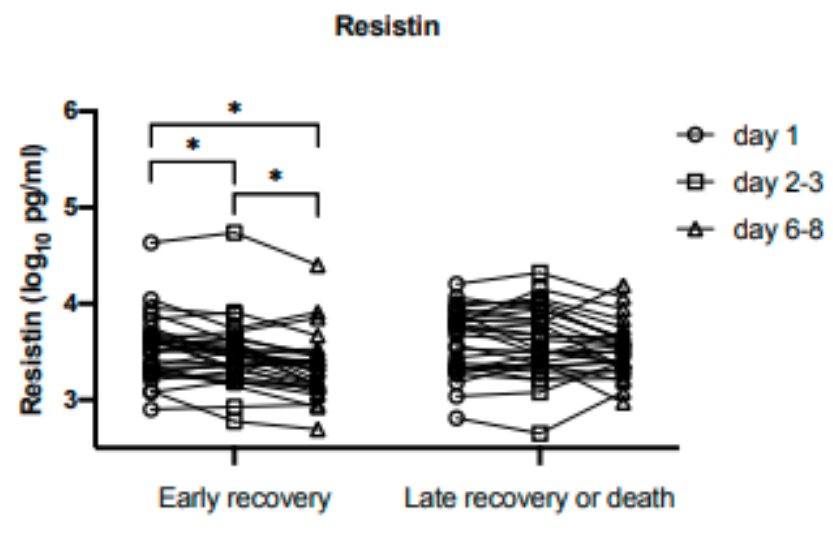

\section{Figure 3}

The relationship between resistin and the time to wean off MV. We defined the day of weaning off MV as the day of extubation for patients without tracheostomy or the day ventilator withdrawal for patients with tracheostomy. Resistin levels were transformed to common logarithmic values to normalize the data distribution. All data are expressed as the mean \pm SE. (A) The resistin levels in the early recovery patients (weaning off $M V \leq 12$ days) and patients with late recovery ( $>12$ days) or death on day $1(n=35 ; n=26$, respectively), day 2-3 $(n=34 ; n=25$, respectively), and day 6-8 $(n=32 ; n=26)$ * indicates a statistically significant $(p<0.05)$ difference between early recovery patients and patients with late recovery or death on each day. (B) Each plot represents a sample, and samples from the same case are connected by a line. * indicates a statistically significant difference $(p<0.05)$ by Wilcoxon signed-rank test. MV, mechanical ventilation; SE, standard error 


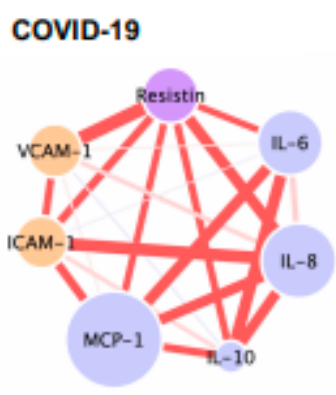

day 1

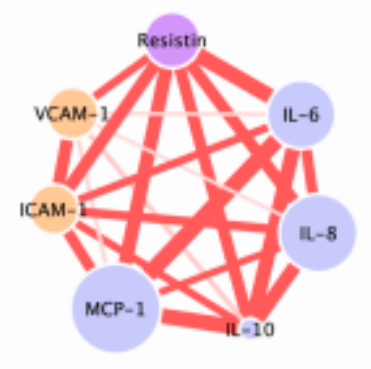

day $2-3$

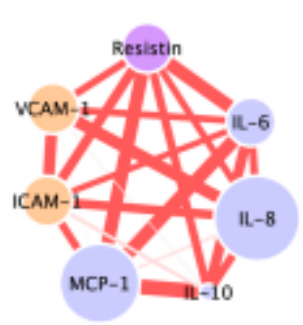

day 6-8

\section{Sepsis}

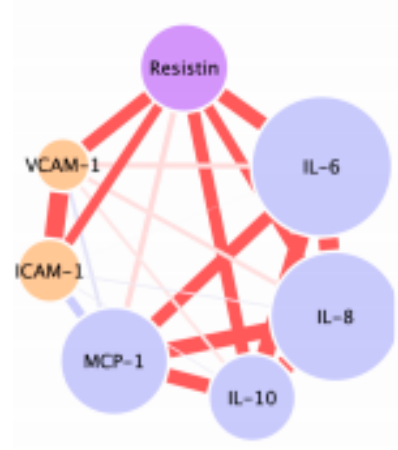

day 1

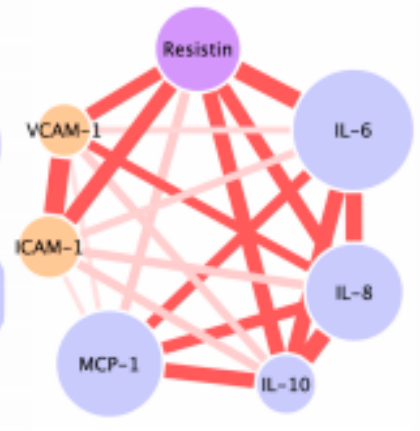

day $2-3$

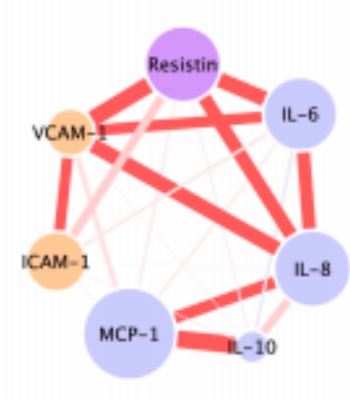

day $6-8$

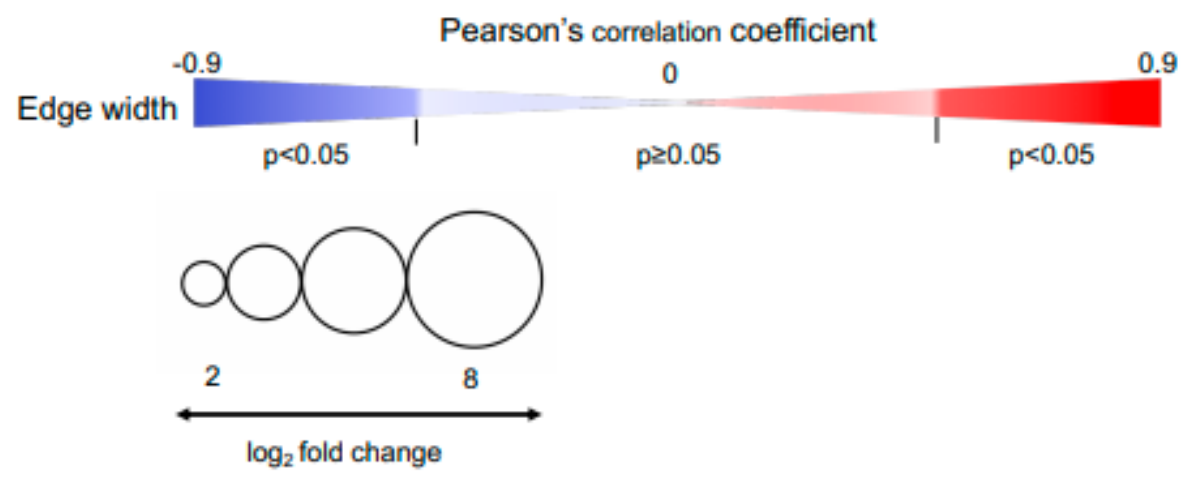

\section{Figure 4}

Network visualization of resistin, 4 cytokines and 2 endothelial damage markers. Resistin, cytokines and endothelial damage markers were transformed to common logarithmic values to normalize the data distribution. The width of each edge indicates the Pearson's correlation coefficients among resistin, cytokines and endothelial damage markers. The red edge indicates a statistically significant correlation. The size of each node was determined based on the log2 fold change (i.e., average resistin, cytokine or endothelial damage marker levels in COVID-19 or sepsis patients/average resistin, cytokines or endothelial damage marker levels in controls). Node colors depict resistin (purple), cytokines (light blue) and endothelial damage markers (orange). COVID-19, coronavirus disease 2019; ICAM-1, intercellular adhesion molecule 1; VCAM-1, vascular cell adhesion molecule-1; IL, interleukin; MCP, monocyte chemotactic protein

\section{Supplementary Files}


This is a list of supplementary files associated with this preprint. Click to download.

- Supplementalfile.docx 